\title{
Linfonodo sentinela no câncer de endométrio: Revisão narrativa
}

\author{
Sentinel lymph node in endometrial cancer: Narrative review \\ Linfonodo centinelaenelcáncer de endometrio: Revisión narrativa
}

Lívia Laura dos Santos Rocha ${ }^{1}$, Daniel Lourenço Lira ${ }^{1}$, Stefano dos Santos Adornoํ․, Glauce Lerina Silva Belém¹, Antônio José Fonseca de Rocha Junior², Moises Castro Saback¹.

\begin{abstract}
RESUMO
Objetivo: Descorrer sobre narrativa do tema linfonodo sentinela no câncer de endométrio. Métodos: Quanto às diretrizes metodológicas, trata-se de uma monografia de revisão narrativa elaborada a partir de pesquisa bibliográfica e referencial teórico publicado sobre a luz do tema. Resultados: Aos resultados, consideraramse os discutidos com o referencial teórico, permitindo considerar que o médico deve confirmar o acometimento da rede linfonodal no CA de endométrio, através de exames complementares, para esse recurso há duas metodologias: Pesquisa de Linfonodo Sentinela (LNS) através da injeção de corantes ou através de injeção de marcador por radioisótopo, para detecção de (LNS) das regiões pélvica ou para-aórtica em CA de endométrio, através de injeção cervical, seguida de detecção por visualização dos linfonodos corados ou com o uso de Gamma Probe ou outro método de detecção dos linfonodos marcados. O exame é o mais acessível, em custos, e pode reduzir a necessidade de extração de linfonodos sadios. Conclusão: Procedimentos de pesquisa de linfonodos sentinelas no câncer de endométrio, muitas vezes, pode ser evitar parcial ou totalmente a linfadenectomia clássica, dependendo para isso da avaliação clínico do especialista, esse procedimento de estadiamento cirúrgico permite intervenções menos agressivas e com baixo custo operacional e rápida recuperação da cliente.
\end{abstract}

Palavras Chaves: Câncer de endométrio, Linfadenectomia, Linfonodos sentinelas do CA de endométrio.

\section{ABSTRACT}

Objective: To review the narrative of the sentinel lymph node theme in endometrial cancer. Methods: As regards the methodological guidelines, it is a monograph of narrative revision elaborated from bibliographical research and theoretical reference published on the light of the subject. Results: The results were considered those discussed with the theoretical reference, allowing to consider that the physician must confirm the involvement of the lymph node network in the endometrial CA by means of complementary tests, for this method there are two methodologies: Sentinel Lymph Node Research (LNS) by dye injection or by radioisotope injection for detection of (LNS) of the pelvic or para-aortic regions in endometrial CA through cervical injection, followed by visual detection of lymph nodes stained or using Gamma Probe or another method of detecting marked lymph nodes. The examination is the most affordable, in costs, and may reduce the need for healthy lymph node extraction. Conclusion: Sentinel lymph node investigation procedures in endometrial cancer can often be partially or totally avoided by classical lymphadenectomy, depending on the clinical evaluation of the specialist, this surgical staging procedure allows for less aggressive interventions with low operational costs and customer recovery.

Keywords: endometrial cancer, lymphadenectomy, endometrial CA sentinel lymph nodes.

${ }^{1}$ Universidade Estadual do Amazonas (UEA), Manaus - AM. *E-mail: Iivinharocha53@hotmail.com

2 Universidade Federal do Amazonas (UFAM), Manaus - AM. 


\section{RESUMEN}

Objetivo: Descurrir sobre la narrativa del tema del ganglio centinela en el cáncer de endometrio. Métodos. En cuanto a las directrices metodológicas, se trata de una monografía de revisión narrativa elaborada a partir de la investigación bibliográfica y referencial teórica publicada sobre la luz del tema. Resultados: A los resultados, se consideraron los discutidos con el referencial teórico, permitiendo considerar que el médico debe confirmar el acometimiento de la red linfonodal en el CA de endometrio, a través de exámenes complementarios, para ese recurso hay dos metodologías: Investigación de Linfonodo Centinela (LNS) a través de la inyección de colorantes oa través de inyección de marcador por radioisótopo, para detección de (LNS) de las regiones pélvica o para-aórtica en CA de endometrio, a través de inyección cervical, seguida de detección por visualización de los ganglios linfáticos colorados o con el uso de Gamma Probe u otro método de detección de los ganglios marcados. El examen es el más accesible, en costos, y puede reducir la necesidad de extracción de ganglios sanos. Conclusión: Los procedimientos de investigación de los ganglios centinela en el cáncer de endometrio, a menudo, pueden ser evitar parcial o totalmente la linfadenectomía clásica, dependiendo para ello de la evaluación clínica del especialista, este procedimiento de estadificación quirúrgica permite intervenciones menos agresivas y con bajo costo operacional y, rápida recuperación de la cliente.

Palabras claves: Cáncer de endometrio, Linfadenectomía, Linfonodos centinelas del CA de endometrio.

\section{INTRODUÇÃO}

A crescente a prevalência do câncer vem apresentado uma expressiva importância no cenário mundial. $O$ câncer de endométrio é o sexto tipo mais comum de câncer em mulheres no mundo e de acordo com o Instituto Nacional do Câncer (INCA), este é o sétimo mais frequente no Brasil, excluindo-se os cânceres de pele não melanoma e representa a quarta causa de morte por câncer em mulheres no país.

Este é o tipo de câncer mais comum nos países desenvolvidos, estando relacionado ao estilo de vida ocidental tendo como fatores de risco a obesidade e sedentarismo. Conforme, a Federação Internacional de Ginecologia e Obstetrícia (FIGO), a maioria das pacientes apresentam-se na maioria das vezes no estádio I estas demonstram prognóstico com taxas de sobrevida em torno de 90\% a 95\%. Ensaios clínicos estão em andamento em pacientes com alto risco de recorrência (incluindo quimioterapia, quimioradioterapia e terapias moleculares) para avaliar as modalidades que melhor equilibram a otimização da sobrevida com os menores efeitos adversos na qualidade de vida (MORICE et al, 2016)

A metástase através da cadeia pélvica e para-aórtica é a principal forma de disseminação desse tumor, a presença de acometimento desses linfonodos é determinante para o seu estádio, tratamento e prognóstico da doença. No tratamento cirúrgico padrão deste tipo de câncer, estão inclusos a linfadenectomia pélvica e para-aórtica clássica, que apresentam morbidades e complicações significativas no pós-operatória.

Exceto em raras circunstâncias em que a cirurgia é contraindicada, o tratamento inicial do câncer de endométrio aparente em estágio inicial (isto é, doença supostamente limitada ao útero) requer estadiamento cirúrgico, que inclui histerectomia, salpingo-ooforectomia bilateral e avaliação envolvimento linfonodal regional, especificamente as cadeias linfonodais pélvicas e aórticas. A decisão de realizar uma dissecção linfonodal e em que extensão (pélvica vs. pélvica e aórtica; abaixo vs. acima da artéria mesentérica inferior; linfadenectomia completa vs. amostragem linfonodal) tem sido uma das áreas mais controversas na gestão de câncer endometrial. Nesse contexto, o mapeamento linfático emergiu recentemente como uma nova estratégia promissora e está sendo cada vez mais adotado pelas práticas de oncologia ginecológica nos EUA e no mundo (FADY et al. 2016). 


\section{MÉTODOS}

O estudo trata-se de uma pesquisa bibliográfica, realizadas por meios de fontes secundárias tais como livros didáticos de saúde, artigos, abordando o tema proposto

A pesquisadora trabalha a partir de contribuições dos autores dos estudos analíticos constantes dos textos. O universo bibliográfico abrangeu livros e artigos científicos, disponibilizados em bancos de dados científicos: Biblioteca Virtual de Saúde (BVS); Scientific Electronic Library Online (SCIELO). Estudo foi realizado a partir de uma pesquisa bibliográfica de método dedutivo e descritivo. Quanto o tipo de abordagem, utilizou-se a qualitativa do tipo exploratória, fazendo referência a fundamentos epistemológicos com objetivo de proporcionar maiores informações sobre o assunto.

\section{RESULTADOS E DISCUSSÃO}

\section{1. $O$ que é o câncer de endométrio}

Para García (2017), embora o endométrio possa proporcionar a alegria da concepção de uma nova vida, também pode sofrer influências externas ou internas e passar a funcionar de forma inadequada. As doenças endometriais podem ser tratadas e possivelmente controladas com uso de hormônios sintéticos, ou em alguns casos, com o tratamento cirúrgico, ambos, devidamente acompanhados pelo profissional médico especializado, o que é essencial para evitar complicações da evolução de cada doença, mantendo assim a saúde uterina, aumentando as chances de gestar e/ou de levar uma vida normal. Entre as principais doenças do endométrio temos o pólipo endometrial, hiperplasia endometrial, adenomiose, endometriose e o câncer de endométrio.

O tipo histológico mais comum de câncer de endométrio é o adenocarcinoma endometrióide, que corresponde entre $75 \%$ a $80 \%$ dos casos, e tem como variante mais comum a diferenciação escamosa. Esse tumor está relacionado ao hiperestrogenismo e a lesão precursora é a hiperplasia atípica. O adenocarcinoma seroso papilífero soma $10 \%$ dos casos e o de células claras $4 \%$; são menos comuns e se assemelham aos do ovário e tuba uterina, podendo apresentar disseminação peritoneal. Esses tumores estão associados à mutação genética p53, ocorrem em mulheres mais velhas, frequentemente são diagnosticados em estádios mais avançados e apresentam pior prognóstico. Os demais tipos histológicos são ainda mais raros: mucinosos, escamosos e indiferenciados. Os adenocarcinomas são agrupados de acordo com o grau de diferenciação histopatológica: - G1 (bem diferenciado), apresentando $5 \%$ ou menos do padrão de crescimento não escamoso; - G2 (moderadamente diferenciado), apresentando $6 \%$ a 50\% do padrão de crescimento não escamoso; e, - G3 (indiferenciado), apresentando mais de 50\% do padrão de crescimento não escamoso (FEBRASGO, 2010)

O fator de risco considerado pela maioria dos autores como mais importante é a obesidade, mas constituem também fatores de risco o diabetes mellitus, todos estados relacionados com ação estrogênica aumentada e/ou persistente por longo tempo como menarca precoce, menopausa tardia, baixa taxa de natalidade ou nuliparidade, tumores ovarianos produtores de hormônios, anovulação, uso de terapia estrogênica e, mais recentemente uso de tamoxifeno.

O principal sintoma é o sangramento uterino anormal. Outros comemorativos relacionados à doença são: sensação de peso em baixo ventre; dor pélvica; menorragia; sangramento intermenstrual; e presença de piometra, hematometra e de células glandulares atípicas no exame colpocitológico. Em fase mais tardia ocorre dor em baixo ventre, secreção com odor fétido, alterações urinárias ou intestinais e emagrecimento.

O diagnóstico precoce é a uma das formas mais eficazes de se prevenir a morte por câncer de endométrio. O diagnóstico normalmente é feito a partir da investigação de pacientes com sangramento uterino anormal, quando são realizados exames de ultrassom e histeroscopia ou curetagem uterina. O diagnóstico definitivo do câncer endometrial é realizado pelo exame histopatológico. 


\section{Técnica de Injeção}

O mapeamento linfático no câncer endometrial seguiu um caminho semelhante desde sua primeira descrição em 1996. O objetivo inicial era investigar se o que se tornaria o padrão de tratamento no câncer de mama e melanoma poderia ser aplicável ao câncer de endométrio, identificando especificamente os linfonodos sentinela (SLNs) inicialmente envolvidos na disseminação metastática de células cancerígenas, tornando a linfadenectomia pélvica e aórtica desnecessárias para fins de estadiamento. Os dois aspectos técnicos que precisavam ser inicialmente avaliados envolviam determinar qual sítio de injeção era o mais adequado para permitir que o corante ou o radiocolóide seguissem a rota de possível disseminação metastática e qual marcador (radiocolóide vs. corante azul) estava associado às maiores taxas de detecção (FADY et al, 2016)

A principal vantagem no mapeamento linfático é a possível identificação de metástases de baixo volume por meio de corte em série e coloração imuno-histoquímica (ultrastaging) dos Nódulos do Sistema Linfático (NSL). As metástases de baixo volume incluem micrometástases e Células Tumorais Isoladas (ICG), semelhantes ao descrito na literatura sobre câncer de mama. Grandes séries de pesquisas têm identificado uma porcentagem significativa de metástases de baixo volume em LNS's submetidos à ultrastaging, no caso de câncer endometrial em estágio inicial, grupo esse que a dissecação clássica dos nódulos linfáticos provavelmente pode ser evitada (STIEPCICH et al, 2017).

Três locais de injeção foram utilizados principalmente: o fundo uterino, o endométrio com histeroscopia e o colo do útero. O local da injeção basal não foi associado a altas taxas de detecção, e muitas vezes não é tecnicamente viável quando há distorção do útero secundária aos leiomiomas, o que não é uma ocorrência incomum. A abordagem histeroscópica enfocou o conceito de injeção peritumoral e envolveu a identificação do tumor por visualização direta e injeção do endométrio subjacente. Os relatórios publicados inicialmente com essa abordagem foram animadores, indicando uma alta taxa de detecção em ambas as cadeias pélvica e aórtica, mimetizando a distribuição das metástases linfonodais, apesar destes resultados iniciais encorajadores, esta técnica não foi amplamente adotada, devido uma abordagem tecnicamente mais envolvida (a visualização histeroscópica do tumor e do endométrio circundante não é fácil quando o tumor é muito grande e ocupa toda a cavidade endometrial, ou muito pequeno / microscópico e mais difícil de identificar e frequentemente envolve um procedimento separado no dia anterior).A terceira abordagem de injeção, que ganhou mais popularidade nos últimos anos, envolve injeção no colo do útero, pois o mesmo é quase sempre claramente visualizado e acessível, apesar da grande distorção do corpo uterino (por tumores ou leiomiomas). Os relatórios nacionais e internacionais mais recentes utilizam principalmente o colo do útero como local de injeção. A principal crítica à abordagem da injeção cervical é a baixa taxa de mapeamento na área aórtica. Outras experiências e refinamento das técnicas podem levar ao desenvolvimento de uma linfadenectomia seletiva baseada na visualização direta da drenagem linfática do útero, em vez da atual amostragem aleatória (BURKE et al, 1996)

\section{Preditores do sucesso: cirurgião, paciente, técnica}

Alguns outros fatores podem influenciar a taxa de detecção, se não forem levados em consideração, podem afetar significativamente os resultados do mapeamento linfático. A experiência do cirurgião é um desses fatores. O número de casos necessários para atingir um certo nível de proficiência em qualquer técnica depende do cirurgião individual, do volume de casos e, provavelmente, da técnica utilizada. É importante para os cirurgiões individualmente estabelecerem as suas próprias taxas de detecção e taxas de falso-negativos (FNRs), independentemente de quantos casos realizaram durante seu treinamento, para determinar precisamente quando podem oferecer esta opção aos seus pacientes, e em quais circunstâncias.

Além dos fatores relacionados à técnica e ao cirurgião, o mapeamento bem sucedido também pode ser afetado por fatores do paciente, por exemplo a obesidade tem sido relatada para afetar mapeamento bem sucedido. Células Tumorais Isoladas (ICG) foi recentemente relatado para melhor desempenho do que 0 corante azul em pacientes obesos. 


\section{Preditores da taxa de falso - negativos}

O objetivo ideal do mapeamento linfático é identificar um ou um número limitado de Linfonodos Sentinela (SLNs) que serão os primeiros envolvidos com a doença metastática e, portanto, representativos do restante da cadeia nodal. Especificamente, um SLN negativo para doença metastática indicaria então com alta confiança (geralmente na faixa de $90 \%$ a $95 \%$ ) que o restante dos linfonodos não está envolvido, tornando desnecessária a dissecção de linfonodos.

Batista et al (2013), define que as metástases em linfonodos é um importante fator prognóstico do câncer de endométrio e colo do útero. A disseminação por via linfática, e como acontece a propagação por meio dos linfonodos, nos remete a inclusão da linfadenectomia pélvica sistemática ao tratamento cirúrgico do câncer de endométrio e cervical em fase inicial, entretanto ainda não dispomos de evidencias suficientemente concretas do benefício dos pacientes em fase inicial do câncer em relação a dissecção sistemática dos linfonodos.

O número de linfonodos dissecados durante a linfadenectomia total, parece trazer benefícios terapêuticos aos pacientes que possuem câncer operável, acredita serque devido ao quantitativo de linfonodos pélvicos retirados durante o procedimento (número de linfonodos pélvicos dissecados - NLPD) possa melhorar 0 estadiamento linfonodal destes pacientes. Atualmente abordagens mais conservadoras e individualizadas vêm sendo adotadas para uma redução da mortalidade durante o tratamento. Segundo INCA, 2017 esses métodos mais atuais avaliam um número menor de linfonodos e o valor clinico do NLPD para estadiamentoto linfonodal do câncer de endométrio, entretanto devido à complexidade e bilateralidade das cadeias linfonodias que drenam o útero, essas técnicas têm se tornado desafiadoras frente a adaptação para o câncer de endométrio (BODURTHA SMITH, et al 2017)No entanto, adaptar seu uso no câncer de endométrio tem sido mais desafiador, dada a complexidade e bilateralidade das cadeias nodais que drenam o útero (BODURTHA SMITH, et al 2017)

Remoção de linfonodos para estadiamento, como parte da cirurgia inicial. É usado no manejo de pacientes com carcinoma endometrial e continua a ser um tema controverso em ginecológica oncológica. (INCA, 2017).

A medida típica que determinará se a disseminação de células metastáticas segue esse padrão previsível é a taxa de falso negativo (FNR) do protocolo de mapeamento linfático. Em outras palavras, a taxa de falso negativo indica a probabilidade de ter células metastáticas em não-linfonodos sentinelas quando o SLN é negativo. Para calcular a taxa de falso negativo com precisão, a população utilizada para este cálculo deveria ter sido submetida à remoção do NLS, bem como não-SLNs nas cadeias regionais em risco. Para o câncer endometrial, o cálculo de uma taxa de falso negativo precisa exigiria a remoção dos SLNs e, então, seria realizada uma dissecção sistêmica dos linfonodos pélvicos e aórticos. Na ausência deste, teoricamente, podese concluir que o mapeamento linfático não deve ser usado para o estadiamento do câncer endometrial antes que uma FNR confiável seja estabelecida. No entanto, isso seria uma conclusão prematura se não analisarmos os dados atuais e analisarmos os benefícios do mapeamento linfático, reconhecendo as peculiaridades do câncer endometrial. Nesse aspecto, qualquer discussão sobre o papel atual e o benefício do mapeamento linfático não seria precisa se não fosse feita dentro do contexto de discutir o papel da dissecção linfonodal no câncer endometrial, que é a maior questão de controvérsia que o mapeamento linfático está tentando resolver.

A questão, portanto, não é meramente se o mapeamento linfático está associado a taxas de falso negativos muito baixos, mas também se uma estratégia de mapeamento linfático é mais benéfica do que uma estratégia de linfadenectomia completa, mesmo associada a um FNR maior do que o aceitável em outros tumores sólidos.

O Memorial Sloan Kettering Cancer Center LNS algoritmo pode ser usado como lista de verificação para garantir a padronização dos cuidados e para reduzir a chance de doença nodal. Prospectivamente estão em curso ensaios em muitas instituições para ajudar a estabelecer, o papel definitivo do mapeamento de LNS 
para estadiamento do câncer endometrial. Com o objetivo de fornecer uma atualização sobre os dados clínicos mais recentes relacionados ao Mapeamento linfático visando o estadiamento e gerenciamento do Câncer de endométrio em sua prática clínica (FADY, 2016).

Embora uma baixa taxa de falso negativo seja necessária para concluir que o mapeamento do SLN possa substituir com confiança a linfadenectomia pélvica e aórtica completa, a controvérsia em torno do papel da linfadenectomia pélvica e aórtica no câncer endometrial permitiu que o mapeamento linfático se tornasse uma alternativa aceitável, mesmo na ausência de dados prospectivos para calcular o FNR.

Segundo (BODURTHA SMITH, et al 2017) em análise recente sugere se que a taxa de detecção do linfonodo sentinela e a sensibilidade do câncer endometrial aproximam-se daquelas observadas no câncer de mama e no melanoma, malignidades nas quais o mapeamento do NLS é o padrão de atendimento.

\section{Avaliação dos Linfonodos Aórticos: Obrigatórios ou Opcional?}

Uma parte importante da controvérsia sobre a avaliação do linfonodo sentinela no estadiamento cirúrgico do câncer de endométrio diz respeito à avaliação dos linfonodos paraorticos. Existe pouco consenso de que a realização de uma dissecção completa dos linfonodos aórticos até o nível das veias renais identifique uma proporção significativa de pacientes com metástases nas cadeias aórticas. A questão, no entanto, é se a identificação e remoção desses linfonodos envolvidos está associada à melhora da sobrevida, seja diretamente como resultado de sua remoção cirúrgica ou permitindo um tratamento adjuvante mais adequado.

Para mapeamento linfático, o sítio cervical de injeção é o mais comumente usados em relatórios publicados. A crescente experiência no mapeamento linfático de diferentes grupos parece indicar que uma vez que um certo nível de competência seja alcançado, uma taxa muito alta de mapeamento pode ser alcançada na pelve. No entanto, o mapeamento da área aórtica tem tido menos sucesso. Embora SLNs possam ser identificados na área aórtica após uma injeção cervical, esta é uma ocorrência pouco frequente, não ultrapassando $5 \%$.

Segundo estudos atuais, (BIOCCHI et al, 2017) a maioria dos pacientes com câncer endometrial pode seguramente serem poupados da dissecção dos linfonodos para-aórticos, independentemente do tipo histológico. Estes podem ser selecionados pela combinação de ausência de metástases em linfonodos pélvicos e ausência de invasão profunda do miométrio. Quando somente a avaliação da profundidade de comprometimento miometrial pode ser avaliado, em casos de comprometimento superficial do miométrio (<50\%) menos de $1 \%$ terão metástases em linfonodos para-aórticos.

\section{Papel do Ultrastaging}

Uma das vantagens potenciais do mapeamento linfático é a possibilidade de identificar metástases de baixo volume por seccionamento serial adicional e coloração imuno-histoquímica (ultrastaging) do NLS. Metástases de baixo volume incluem micrometástases e células tumorais isoladas (ITCs), semelhantes às descritas na literatura sobre câncer de mama. Grandes séries identificaram uma porcentagem significativa de metástases de baixo volume em SLNs submetidos a ultrastaging, incluindo em pacientes com câncer de endométrio de baixo grau, em estágio inicial, um grupo no qual a dissecção de linfonodos indiscutivelmente poderia ter sido omitida.

Nesse momento, o significado clínico dessas metástases de baixo volume é desconhecido, assim como o tratamento pós-cirúrgico ideal quando identificado. Optar por ignorá-las é difícil na ausência de dados clínicos tranquilizadores, e tratá-los como macrometástases pode submeter o paciente às complicações do tratamento adjuvante desnecessariamente, especialmente quando essas metástases de baixo volume são identificadas em pacientes de baixo risco com resultados tipicamente excelentes.

A controvérsia em torno do valor do ultrastaging no câncer endometrial, o que reflete a incerteza mais ampla sobre o valor da avaliação linfática no câncer endometrial. Embora estudos prospectivos randomizados demonstrem o impacto limitado da linfadenectomia na sobrevida do paciente, dados retrospectivos sugerem 
um benefício terapêutico da linfadenectomia em pacientes de alto risco. Assim, o verdadeiro valor do mapeamento do linfonodo sentinela pode ser a adaptação da terapia adjuvante para pacientes de alto risco, além de minimizar o risco de dano que ocorre com a linfadenectomia total (FROST et al 2015).

\section{CONCLUSÃO}

O carcinoma de endométrio é o câncer ginecológico mais comum do mundo desenvolvido. A maioria das mulheres apresenta-se no estádio I da doença, com bom prognóstico e taxa de sobrevida global superior a $90 \%$. O tratamento definitivo para o carcinoma endometrial é a histerectomia total abdominal e salpingoooforectomia bilateral associada ou não à linfadenectomia pélvica e para-aórtica. A relevância da dissecção linfonodal e da adjuvância permanece controversa particularmente na doença inicial, dada a excelente taxa de sobrevida global dessas pacientes e as possíveis sequelas associadas a linfadenectomia clássica. Este trabalho permite concluir que, seguindo um protocolo de segurança, o mapeamento do linfonodo sentinela no estadiamento do câncer de endométrio é uma possível e segura alternativa a dissecção linfonodal completa.

\section{REFERÊNCIAS}

1. BAIOCCHI, Glauco, et al. O impacto no nódulo linfático sentinela (SLN) - mapeamento na implantação de câncer de endométrio de alto risco. Departamento de Oncologia Ginecológica, AC Camargo Câncer Center, São Paulo, Brasil. Sociedade de Oncologia Cirúrgica, 2017.

2. BATISTA, Thales Paulo, et al. Qual a importância do número de linfonodos pélvicos dissecados para o estadiamento locorregional do câncer de colo uterino? Hospital do Câncer de Pernambuco, Recife, PE, novembro de 2013.

3. BODURTHA SAJ, et al.Sentinel lymph node assessment in endometrial cancer: a systematic review and meta-analysis. American Journal of Obstetrics \& Gynecology , Volume 216 , Issue 5 , 459 - 476.2017.

4. BURKE TW, et al. Intra abdominal Lymphatic Mapping to Direct Selective Pelvic and Paraaortic Lymphadenectomy in Women with High- Risk Endometrial Cancer: Results of a Pilot Study. Gynecologic Oncology, Volume 62 , Issue 2, 169 - 173.1996.

5. FADY KC, et al. Divisão de Oncologia e Ginecológica, Maimonides Medical Center, Brooklyn. Nova York, EUA, maio de 2016.

6. FEBRASGO. Federação Brasileira das Associações de Ginecologia e Obstetrícia.Manual de Orientação Ginecologia Oncológica. 2010.

7. FROST, et al. Linfadenectomia para o tratamento do câncer endometrial. Dados Cochrane Syst Rev. 2015

8. GARCÍA, Marina Martínez. O endométrio. Revista Eletrônica Infoescola Navegando e Aprendendo, setembro de 2017. Disponível em: http://www.infoescola.com/histologia/endometrio/.

9. INCA, Instituto Nacional de Câncer. Protocolos de atendimento de CA Disponível em: $<$ http://www1.inca.gov.br/vigilancia/incidencia.asp>.

10.MORICE P, et al. E. Câncer endometrial. Lancet (Londres, Inglaterra) ; 387 : 1094-1108. 2016.

11.STIEPCICH, M; et al. Adenocarcinoma do endométrio. Revista Eletrônica Educação Médica Fleury $\begin{array}{lllll}\text { Medicina } & \text { S Maúde. } & 2017 . & \text { Disponível }\end{array}$ em:<http://www.fleury.com.br/medicos/educacaomedica/revistamedica/materias/Pages/adenocarcinoma-doendometrio.aspx>. 